\title{
Higgs inflation and general initial conditions
}

\author{
Sarang Zeynizadeh ${ }^{1,2, a}$, Amin Rezaei Akbarieh ${ }^{3,4, b}$ \\ ${ }^{1}$ Department of Physics, Sharif University of Technology, Tehran, Iran \\ 2 School of Physics, Institute for Research in Fundamental Sciences (IPM), Tehran, Iran \\ ${ }^{3}$ Research Institute for Astronomy and Astrophysics of Maragha (RIAAM), 55134-441 Maragha, Iran \\ ${ }^{4}$ Department of Theoretical Physics, Tabriz University, Tabriz, Iran
}

Received: 10 February 2015 / Accepted: 14 June 2015 / Published online: 4 August 2015

(C) The Author(s) 2015. This article is published with open access at Springerlink.com

\begin{abstract}
A Higgs field of particle physics can play the role of the inflaton in the early universe if it is non-minimally coupled to gravity. The Higgs inflation scenario predicts a small tensor to scalar ratio: $r \simeq 0.003$. Although this value is consistent with the upper bound $r<0.12$ given by the BICEP2/Keck Array and Planck data, it is not at their maximum likelihood point: $r \simeq 0.05$. Inflationary observables depend not only on the inflationary models, but they also depend on the initial conditions of inflation. Changing the initial state of inflation can improve the value of $r$. In this work, we study the Higgs inflation model under general initial conditions and show that there is a subset of these general initial conditions which leads to enhancement of $r$. Then we show that this region of parameter space is consistent with a non-Gaussianity bound.
\end{abstract}

\section{Introduction}

The inflationary epoch of the early universe has became an important part of the standard big bang model of cosmology [1]. The inflationary paradigm not only solves two basic problems of standard cosmology, i.e. the horizon and flatness problems, but it also predicts that the large-scale structure of universe originating from the primordial perturbation is nearly scale invariant, which is in good agreement with observations [2,3].

In single field models of inflation generally a scalar field, which is called inflaton, drives an exponential expansion. It will be economical if we identify a known particle with the inflaton field. The Higgs field of particle physics might be an opportunity for this identification with the inflaton field. The first model constructed on this assumption has been proposed by Bezrukov and Shaposhnikov [7]. They claimed that

\footnotetext{
a e-mail: zeynizadeh@physics.sharif.ir

be-mail:am_rezaei@physics.sharif.ir
}

the Higgs field can be identified with the inflaton field if it is non-minimally coupled to gravity. At the same time, the dimensionless coupling constant $\xi$, which will be defined in Sect. 2, should be of the order of $10^{4}$. This large value of the coupling constant leads to unitarity violation [10-15]. Unitarity violation implies that there should be a UV cut off $\Lambda$. Beyond $\Lambda$ our effective theory will break down. Hence our theory should be replaced by a new fundamental theory beyond the UV cut off. Another issue related to the model in [7] is the very small value prediction for the tensor to scalar perturbation ratio $r$, i.e. $r \simeq 0.003$. The announcement of BICEP2 for B-mode detection [3] with a large value $r \simeq 0.2$ motivated people to make some efforts to reconcile Higgs inflation with the BICEP2 results [16-19]. Soon, it turned out that there is serious doubt about the BICET2 results $[4,5]$. Recently, Keck Array claimed that they also have found an excess of B-mode power over the standard expectation which is consistent with the BICEP2 results [6]. In a joint analysis, BICEP2/Keck Array and Planck collaborations report their results as a likelihood curve for $r$ with an upper limit $r<0.12$ and a maximum likelihood at $r \simeq 0.05$ [8]. Although the predicted value for $r$ by the Higgs inflation model is consistent with the upper limit in [8], it still is far enough from the maximum likelihood $r \simeq 0.05$. Therefore, it is reasonable to search for some way to increase the $r$ value. ${ }^{1}$ In the presence of a UV cut off, which in turn introduces new physics, $r$ can be altered due to the non-trivial initial state effects. Hence, $r \simeq 0.003$ is not a firm prediction of Higgs inflation model, but it will depend on the initial state of inflation.

Determination of the initial conditions is a necessary condition to describe the dynamics of a given system. When there is no UV cut off, the initial condition is trivial and

\footnotetext{
${ }^{1}$ It should be observed that the statistical significance of $r=0.05$ is rather low and it cannot be considered as a detection of primordial tensors. Indeed, in the words of [9], the observations are still "consistent with $r=0$ ".
} 
choosing a Bunch-Davies vacuum satisfied the Minkowski space limit. In the presence of a UV cut off, the effects of the new theory can be set on the non-trivial initial condition or the non-Bunch-Davies vacuum [20,21]. Here we shall show that, by suitable choice of initial conditions, it is possible to get a sizable value for $r$. Recently, Ashoorioon et al. [22,23] employed non-trivial initial conditions for the chaotic model of inflation to suppress the value of $r$ to bring about reconciliation of the Planck data with that of the BICEP2. They excluded a large piece of parameter space by using the observational bound of the non-Gassianity. But in the case of the Higgs inflation model this exclusion does not need to occur due to the special property of the Higgs inflation model. Incidentally, using this region leads to the enhancement of $r$.

The paper is organized as follows. In Sect. 2 we give a brief review of Higgs inflation model proposed in [7]. In Sect. 3, in a very concise review of perturbation theory in cosmology, we just mention the effects of the general initial conditions on the inflationary parameters. In Sect. 4 we argue that for some region of parameter space, we obtain a sizable value for $r$. Finally we discuss the results and summarize them.

\section{Review of Higgs inflation}

To write the standard model of particle physics in the presence of gravity, the Higgs inflation model is one of our choices. In this model the Higgs field is non-minimally coupled to gravity via a dimensionless coupling constant $\xi$ [7]:

$L=L_{S M}-\frac{1}{2} M^{2} R-\xi H^{\dagger} H R$,

where $M$ is some mass scale, $R$ and $H$ denote the Ricci scalar and Higgs field, respectively. The potential term is required by the renormalizibility of the scalar field in a curved background. By choosing a unitary gauge $H=h / \sqrt{2}$, the scalar sector is non-minimally coupled to gravity:

$S_{J}=\int \mathrm{d}^{4} x \sqrt{-g}\left[-\frac{1}{2} M^{2} R-\frac{1}{2} \xi h^{2} R+\frac{1}{2} \partial_{\mu} h \partial^{\mu} h-V(h)\right]$,

where the sub-index $J$ indicates a Jordan frame, and the potential, $V(h)$, is defined by

$V(h)=\frac{1}{4} \lambda\left(h^{2}-v^{2}\right)^{2}$,

where $v=\langle h\rangle$. For $1 \ll \xi \ll 10^{17}$, we can assume $M \simeq M_{p}$ where $M_{p}$ is the reduced Planck mass. Due to the presence of the non-minimal coupling term, it is very cumbersome to work with. By the following conformal transformation, we can transform the Jordan frame to an Einstein frame:

$g_{\mu \nu} \rightarrow \hat{g}_{\mu \nu}=\Omega^{2} g_{\mu \nu}, \quad \Omega^{2}=1+\frac{\xi h^{2}}{M_{p}^{2}}$.
This transformation leads to a non-canonical kinetic term which can be converted to canonical form by the field redefinition

$\frac{d \chi}{d h}=\sqrt{\frac{\Omega^{2}+6 \xi^{2} h^{2} / M_{p}^{2}}{\Omega^{4}}}$.

The action in the Einstein frame becomes

$S_{E}=\int \mathrm{d}^{4} x \sqrt{-g}\left\{-\frac{1}{2} M_{p}^{2} \hat{R}+\frac{1}{2} \partial_{\mu} \chi \partial^{\mu} \chi-U(\chi)\right\}$

where $\hat{R}$ is a Ricci scalar in terms of $\hat{g}_{\mu \nu}$. The potential term $U(\chi)$ is

$U(\chi)=\frac{1}{\Omega^{4}} \frac{\lambda}{4}\left(h(\chi)^{2}-v^{2}\right)^{2}$.

According to (4) and (5), for large values of $h$, i.e. $h \gg$ $M_{p} / \sqrt{\xi}$, we have

$h \simeq \frac{M_{p}}{\sqrt{\xi}} \exp \left(\frac{\chi}{\sqrt{6} M_{p}}\right)$

$U(\chi)=\frac{\lambda M_{p}^{4}}{4 \xi^{2}}\left(1+\exp \left(-\frac{2 \chi}{\sqrt{6} M_{p}}\right)\right)^{-2}$,

and for large values of $h$ or $\chi \gg \sqrt{6} M_{p}$ the potential $U(\chi)$ is flat. Here the Higgs field drives inflation. In order to show whether this potential can give a consistent inflationary expansion, we use the standard slow-roll formalism in the Einstein frame,

$\epsilon=\frac{1}{2} M_{p}^{2}\left(\frac{U^{\prime}}{U}\right)^{2}, \quad \eta=M_{p}^{2} \frac{U^{\prime \prime}}{U}$,

$N=\int \frac{1}{\sqrt{2 \epsilon}} \frac{\mathrm{d} \chi}{M_{p}^{2}}, \quad n_{s}=1-6 \epsilon+2 \eta, \quad r=16 \epsilon$,

where $\epsilon$ and $\eta$ are slow-roll parameters, $N$ is the number of e-foldings, $n_{s}$ denotes the spectral index and $r$ is the tensor to scalar perturbation ratio. Substituting (9) in (10) and considering large field values for $h$ leads to [24]

$\epsilon \simeq \frac{4 M_{p}^{4}}{3 \xi^{2} h^{4}}$

$\eta \simeq-\frac{4 M_{p}^{2}}{3 \xi h^{2}}$,

$N \simeq \frac{6}{8} \frac{\xi}{M_{p}^{2}}\left(h_{N}^{2}-h_{\mathrm{end}}^{2}\right)$,

where $h_{N}$ denotes the field value at the horizon exit and $h_{\mathrm{end}}$ denotes the field value at the end of inflation. The end of inflation corresponds to $\epsilon=1$. Using Eq. (11) we obtain $h_{\text {end }} \simeq \frac{1.07 M_{p}}{\sqrt{\xi}}$. The $N$ is determined from the CMB observation: $N \simeq 57.7$ [24]. Substituting this value in (13) leads to $h_{N} \simeq \frac{9.14 M_{p}}{\sqrt{\xi}}$. From the observation [25] we can put a constraint on the amplitude of the scalar power spectrum, 
$\Delta_{\mathcal{R}}^{2}=\frac{1}{8 \pi^{2}} \frac{H^{2}}{\epsilon M_{p}^{2}} \simeq 2 \times 10^{-9}$.

This constraint can be used to determine the unknown parameter $\xi$. By using (11) and (14) in the slow-roll regime, $U \simeq 3 M_{p}^{2} H^{2}$, we obtain

$\frac{U}{\epsilon}=24 \pi^{2} M_{p}^{4} \Delta_{\mathcal{R}}^{2} \simeq\left(0.027 M_{p}\right)^{4}$.

Evaluating $\frac{U}{\epsilon}$ at $h_{N}$, Eq. (15) gives rise to [24]

$\xi=47000 \sqrt{\lambda}$.

Using (13), $n_{s}$ and $r$ evaluated at $h_{N}$ can be approximated as

$n_{s} \simeq 1-8 \frac{4 N+12}{(4 N+3)^{2}}, \quad r \simeq \frac{192}{(4 N+3)^{2}}$.

Here $N \simeq 57.7$ gives $n_{s} \simeq 0.967$ and $r \simeq 0.0031$ [24].

\section{Primordial perturbation with general initial condition}

In this section we will review the cosmological perturbation theory to realize how the effects of general initial conditions come into the game. We will just mention their effects on inflationary quantities such as the power spectrum, the spectral index, etc. In order to derive the equations governing the perturbation, we consider a minimally coupled scalar field with arbitrary potential [26]

$S=\int \mathrm{d}^{4} x \sqrt{-g}\left[\frac{1}{2} M_{p}^{2} R-\frac{1}{2} g^{\mu \nu} \partial_{\mu} \phi \partial_{\nu} \phi-V(\phi)\right]$.

Perturbations are defined around the homogeneous background given by the solutions of the action (18), i.e. $\bar{\phi}(t)$ and $\bar{g}_{\mu \nu}(t)$

$\phi(t, \boldsymbol{x})=\bar{\phi}(t)+\delta \phi(t, \boldsymbol{x})$,

$g_{\mu \nu}(t, \boldsymbol{x})=\bar{g}_{\mu \nu}(t)+\delta g_{\mu \nu}(t, \boldsymbol{x})$,

and the perturbed metric is parametrized as

$d s^{2}=a^{2}(\tau)\left[-(1+2 \Phi) d \tau^{2}+\left((1-2 \Psi) \delta_{i j}+h_{i j}\right) d y^{i} d y^{j}\right]$.

Here $\tau$ is the conformal time, $a$ is the scalar factor of the FRW metric, $\Phi$ and $\Psi$ are Bardeen potentials, and $h_{i j}$ denotes a symmetric tensor with $h_{i}^{i}=0, \partial^{i} h_{i j}=0$. In addition to physical degrees of freedom, these perturbations also may contain the fictitious gauge freedom. To avoid this gauge freedom, it is useful to introduce a new gauge invariant scalar quantity,

$\mathcal{R}=\Psi \frac{H}{\dot{\bar{\phi}}} \delta \phi$.
This is called a comoving curvature perturbation. Expanding the action in (18) up to second order in terms of $\mathcal{R}$ leads to

$S_{(2)}=\frac{1}{2} \int \mathrm{d}^{4} x a^{3} \frac{\dot{\phi}^{2}}{H^{2}}\left[\dot{\mathcal{R}}^{2}-a^{-2}\left(\partial_{i} \mathcal{R}\right)^{2}\right]$.

By defining the Mukanov-Sasaki variable

$v \equiv z \mathcal{R}, \quad z^{2} \equiv a^{2} \frac{\dot{\phi}^{2}}{H^{2}}$,

the equation of motion corresponding with the second order action becomes

$v_{k}^{\prime \prime}+\left(k^{2}-\frac{z^{\prime \prime}}{z}\right)=0$.

Here $v_{k}$ is the Fourier mode of $v$ and a prime indicates the derivative with respect to conformal time. For a quasi-de Sitter background in the slow-roll limit, the general solution of Eq. (24) can be written as

$v_{k} \simeq \frac{\sqrt{\pi|\tau|}}{2}\left[\alpha_{k}^{S} H_{\frac{3}{2}}^{(1)}(k|\tau|)+\beta_{k}^{S} H_{\frac{3}{2}}^{(2)}(k|\tau|)\right]$.

Here $H_{\frac{3}{2}}^{(1)}$ and $H_{\frac{3}{2}}^{(2)}$ are the Hankel functions of the first and second kind, respectively. $\alpha_{k}^{S}$ and $\beta_{k}^{S}$ are Bogoliubov coefficients that satisfy the Wronskian constraint

$\left|\alpha_{k}^{S}\right|^{2}-\left|\beta_{k}^{S}\right|^{2}=1$.

Since $\alpha_{k}^{S}$ and $\beta_{k}^{S}$ are arbitrary up to the Wronskian constraint, they correspond to the general initial condition. In the case of $\alpha_{k}^{S}=1$ and $\beta_{k}^{S}=0, v_{k}$ corresponds to the standard BD vacuum. The states with generic values of $\alpha_{k}^{S}$ and $\beta_{k}^{S}$ usually are called non-BD vacuum or $\alpha$-vacua. The dimensionless scalar power spectrum is defined by

$\Delta_{S}^{2}=\frac{k^{3}}{2 \pi^{2}}\left|\frac{v_{k}}{z}\right|_{k=a H}^{2}$.

Substituting (25) into (27) leads to [22,23,27]

$\Delta_{S}^{2}=\frac{1}{8 \pi^{2} \epsilon}\left(\frac{H}{M_{p}}\right)^{2} \gamma_{S}, \quad \gamma_{s}=\left|\alpha_{k}^{S}-\beta_{k}^{S}\right|_{k=a H}^{2}$.

The non-Gaussianity is an important probe of the early universe encoded in the bispectrum. Having a power spectrum in the squeezed $k_{3} \ll k_{1} \sim k_{2}$ limit suffices to obtain the bispectrum. According to [28], the three point function of the scalar perturbation in the squeezed limit for $\alpha$-vacua is given by

$$
\begin{aligned}
& \left\langle\mathcal{R}_{\boldsymbol{k}_{1}} \mathcal{R}_{\boldsymbol{k}_{2}} \mathcal{R}_{\boldsymbol{k}_{3}}\right) \simeq(2 \pi)^{3} \delta\left(\boldsymbol{k}_{1}+\boldsymbol{k}_{2}+\boldsymbol{k}_{3}\right) \\
& \quad \times\left[4 \epsilon\left(\frac{k_{1}}{k_{3}}\right) \Phi\left(k_{1}, k_{3}\right)-6 \epsilon+2 \eta\right] P_{\mathcal{R}}\left(k_{1}\right) P_{\mathcal{R}}\left(k_{3}\right) .
\end{aligned}
$$


Here $P_{\mathcal{R}}(k)=\frac{2 \pi^{2}}{k^{3}} \Delta_{S}^{2}$ and

$\Phi\left(k_{1}, k_{3}\right)=2 \operatorname{Re}\left[\alpha_{k_{1}}^{S} \beta_{k_{1}}^{S}\left(\frac{\alpha_{k_{1}}^{S *}-\beta_{k_{1}}^{S *}}{\alpha_{k_{1}}^{S}-\beta_{k_{1}}^{S}}\right)\left(\frac{\alpha_{k_{3}}^{S}+\beta_{k_{3}}^{S}}{\alpha_{k_{3}}^{S}-\beta_{k_{3}}^{S}}\right)\right]$.

Noting Eq. (29), the local non-Gaussianity parameter $f_{\mathrm{NL}}^{\text {local }}$ becomes $^{2}$

$f_{\mathrm{NL}}^{\text {local }} \simeq \frac{5}{12}\left[4 \epsilon\left(\frac{k_{1}}{k_{3}}\right) \Phi\left(k_{1}, k_{3}\right)-6 \epsilon+2 \eta\right]$.

In this paper, since we are only interested in the local configuration of non-Gaussianity, we do not need to consider flattened and equilateral configurations. Similarly, for tensor perturbations, we obtain the following mode function:

$h_{k}(\tau) \simeq \frac{\sqrt{\pi|\tau|}}{2}\left[\alpha_{k}^{T} H_{\frac{3}{2}}^{(1)}(k|\tau|)+\beta_{k}^{T} H_{\frac{3}{2}}^{(2)}(k|\tau|)\right]$.

The dimensionless tensor power spectrum has a similar relation $[22,23,27]$,

$\Delta_{T}^{2}=\frac{2}{\pi^{2}}\left(\frac{H}{M_{P}}\right)^{2} \gamma_{T}, \quad \gamma_{T}=\left|\alpha_{k}^{T}-\beta_{k}^{T}\right|_{k=a H}^{2}$.

Here $\alpha_{k}^{T}$ and $\beta_{k}^{T}$ are Bogoliubov coefficients and satisfy the Wronskian condition $\left|\alpha_{k}^{T}\right|^{2}-\left|\beta_{k}^{T}\right|^{2}=1$. The tensor to scalar perturbation ratio $r$ in the case of $\alpha$-vacua is given by [22,23]

$r=\frac{\Delta_{T}^{2}}{\Delta_{S}^{2}}=16 \epsilon \gamma, \quad \gamma=\frac{\gamma_{T}}{\gamma_{S}}$.

\section{Enhancement of $\boldsymbol{r}$ for some $\alpha$-vacua}

According to (34) for a given $\epsilon \ll 1$, to have sizable value of $r$, it is required that $\gamma \gg 1$. If we would like to have a large value of $r$, we have to search for some special regions in the parameter space of the initial conditions, such that their corresponding $\gamma$, satisfies $\gamma \gg 1$. In the case of the Higgs inflation model with a small $r$, we can use some special initial conditions with $\gamma \gg 1$ to raise the $r$ to a large value. Due to the arbitrariness of $\alpha_{k}$ and $\beta_{k}$ up to the Wronskian condition, we might think that it is possible to obtain a $\gamma$ as large as we would like. In fact, there is an upper limit on the value of $\gamma$ due to the constraints from the back reaction effects and the observational bound on the non-Gaussianity.

Back reaction effects should be small enough not to destroy the inflationary background. By assuming the crude model $\beta_{k} \sim \beta_{0} e^{-\frac{k^{2}}{M^{2} a^{2}}}$, this condition gives rise to [30]

$\left|\beta_{0}\right| \leq \sqrt{\epsilon|\eta|} \frac{M_{p} H}{M^{2}}$

${ }^{2}$ For the definition of $f_{\mathrm{NL}}^{\text {local }}$ refer to [26].
Here we assumed $\epsilon \ll \eta$, which is a reasonable assumption in the case of Higgs inflation. $M$ in (35) is an energy scale of the new physics and our effective theory is valid only at energies lower than the energy scale $M$. In order to convert the constraint on $\beta_{0}$ in (35) to a constraint on $\gamma$, we are following the notation of $[22,23]$. It is mentioned in [22,23] that $\gamma_{S}$ and $\gamma_{T}$ depend on the relative phases of $\alpha_{k}$ and $\beta_{k}$. Therefore, it is useful to parametrize them as

$\alpha_{k}^{S}=\cosh \chi_{S} e^{i \varphi_{S}}, \quad \beta_{k}^{S}=\sinh \chi_{S} e^{-i \varphi_{S}}$,

$\alpha_{k}^{T}=\cosh \chi_{T} e^{i \varphi_{T}}, \quad \beta_{k}^{T}=\sinh \chi_{T} e^{-i \varphi_{T}}$.

Consistency of the above parametrization with $\beta_{k} \sim$ $\beta_{0} e^{-\frac{k^{2}}{M^{2} a^{2}}}$ implies that

$\left|\beta_{0}\right| e^{-\frac{k^{2}}{M^{2} a^{2}}}=\sinh \chi$.

Below the energy scale of new physics, $k<a M$, $e^{-\frac{k^{2}}{a^{2} M^{2}}}$ $\simeq 1$, and (38) becomes

$\left|\beta_{0}\right| \simeq \sinh \chi$

In the Higgs inflation model, (11) and (12) result in $\epsilon \simeq$ $1.8 \times 10^{-4}$ and $\eta \simeq-1.6 \times 10^{-2}$. Thus, using $\Delta_{S}^{2} \simeq 2 \times 10^{-9}$ in (28) gives $\frac{H}{M_{p}}=5.3 \times 10^{-6} / \sqrt{\gamma_{S}}$. Substituting this result into (35) and using (39) lead to

$\frac{M^{2}}{H^{2}} \lesssim 323 \frac{\sqrt{\gamma_{S}}}{\sinh \chi_{S}}$.

There is a similar expression for the tensor modes, except that $\chi_{S}$ is replaced by $\chi_{T}$. Physical expectations imply $M>H$. Let us write $\gamma$ in terms of the new parametrization in (36) and (37):

$\gamma=\left|\frac{\cosh \chi e^{i \varphi_{T}}-\sinh \chi e^{-i \varphi_{T}}}{\cosh \chi e^{i \varphi_{S}}-\sinh \chi e^{-i \varphi_{S}}}\right|^{2}$.

Here we take $\chi_{S}=\chi_{T}=\chi$ for convenience. According to this formula, $\gamma$ can be bigger or smaller than 1, e.g. for $\chi \simeq 1, \varphi_{S} \simeq 0.01$, and $\varphi_{T} \simeq \frac{\pi}{2}$, we get $\gamma \simeq 70$. In $[22,23]$ it has been shown that for $\chi \gtrsim 1, \varphi_{S} \simeq \frac{\pi}{2}$, and for generic values $\varphi_{T}$, we obtain $\gamma \lesssim 1$. The authors of [22,23] drop the $\varphi_{S}$ 's that satisfy $\varphi_{S} \lesssim \frac{\pi}{10}$ because these values violate the observation bound on the non-Gaussianity. However, as we will show, in the Higgs inflation model, because of the smallness of the slow-roll parameter $\epsilon$, we do not encounter such a violation.

Basically, the enhancement of non-Gaussianity due to the general initial condition can occur only for two types of nonGaussianity: the flattened configuration and the local configuration. In the flattened configuration, $k_{1}+k_{2} \simeq k_{3}$, and enhancement finally disappears due to the effect of projection on the CMB surface [30]. In the local configuration, $k_{3} \ll k_{1}+k_{2}$, there is no cancelation and the enhancement of the non-Gaussianity will survive. The effects of the 


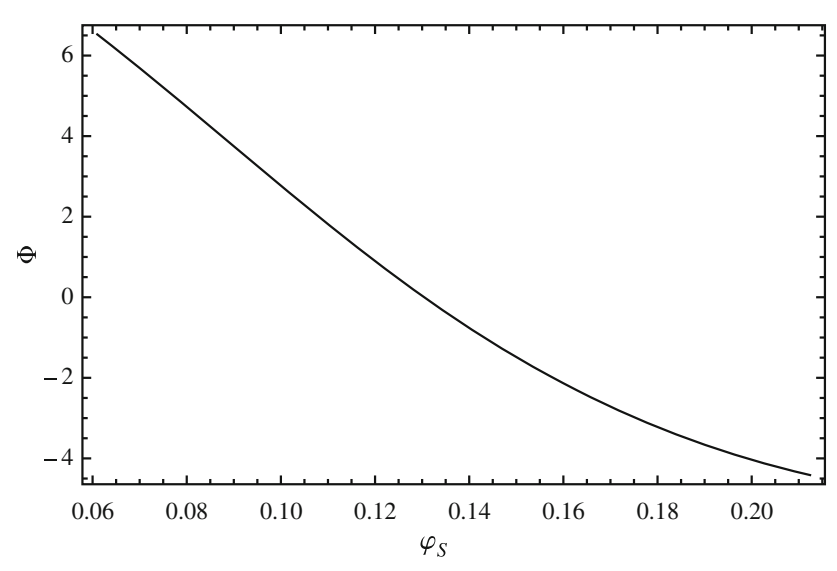

Fig. 1 Diagram of $\Phi$ for $\chi \simeq 0.75$

general initial conditions on the parameter of the local nonGaussianity, i.e. $f_{N L}^{\text {local }}$, are given by $\Phi\left(k_{1}, k_{3}\right)$ in (30). To constrain $\Phi$, let us first write (30) in terms of the parameters introduced in (36),

$$
\begin{aligned}
\Phi\left(k_{1}, k_{3}\right) & =2 \operatorname{Re}\left[\cosh \chi \sinh \chi\left(\frac{\cosh \chi e^{-i \varphi_{S}}-\sinh \chi e^{i \varphi^{S}}}{\cosh \chi e^{i \varphi_{S}}-\sinh \chi e^{-i \varphi_{S}}}\right)\right. \\
& \left.\times\left(\frac{\cosh \chi e^{i \varphi_{S}}+\sinh \chi e^{-i \varphi^{S}}}{\cosh \chi e^{i \varphi_{S}}-\sinh \chi e^{-i \varphi_{S}}}\right)\right] .
\end{aligned}
$$

Here $\sinh \chi\left(k_{3}\right) \simeq \sinh \chi\left(k_{1}\right)$. Because at horizon crossing, $k=a H$, (39) implies sinh $\chi(k) \sim e^{-\frac{H^{2}}{M^{2}}}$, the $k$ dependence of $\sinh \chi(k)$ is lost for $H<M$. Using the Planck data [31],

$-4.2 \lesssim f_{N L}^{\text {local }} \lesssim 5.8$,

and combining (31) and (43) leads to

$-14 \lesssim \Phi \lesssim 19$

Here we take $\frac{k_{1}}{k_{3}} \simeq 10^{2}$ [29]. The smallness of $\epsilon$ in the Higgs inflation model in comparison to its value in other inflationary models (such as the chaotic inflation model, in which $\epsilon \simeq 0.01$ ) allows us to have large values for $\Phi$ in (31), while $f_{N L}^{\text {local }}$ is still in the region specified in (43). The possibility of having a large value of $\Phi$ is equivalent to the possibility of having a very small value for $\varphi_{S}$ (Fig. 1). Small values of $\varphi_{S}$ provide the possibility of reaching large values of $\gamma$. For instance, taking $\varphi_{T} \simeq \frac{\pi}{2}, \varphi_{S} \simeq 0.1$, and $\chi \simeq 0.75$ results in $\gamma \simeq 17, \Phi \simeq 3$ (Fig. 2). These values, by noting (40), lead to $M \simeq 13 H$, consistent with $M>H$. Using $\gamma \simeq 17$ in (34) gives the improved value $r \simeq 0.05$.

It should be noticed that although $\alpha$-vacua as the initial state of the system for some region of parameter space increase the tensor to scalar perturbation ratio, it does not affect the spectral index. The spectral index is defined by

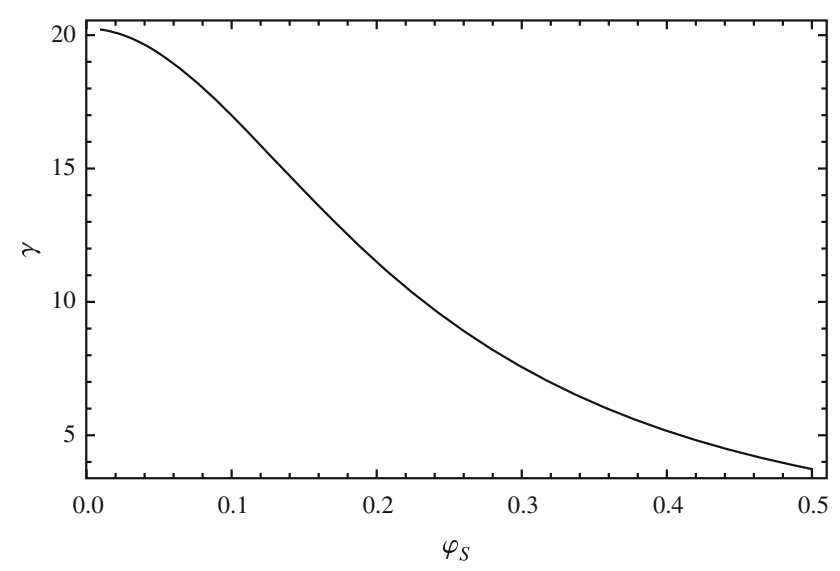

Fig. 2 Diagram of $\gamma$ for $\chi \simeq 0.75$ and $\varphi_{T} \simeq \frac{\pi}{2}$

$n_{S}-1=\frac{\mathrm{d} \ln \Delta_{S}^{2}}{\mathrm{~d} \ln k}=\frac{\mathrm{d}}{\mathrm{d} \ln k}\left[\ln \frac{1}{8 \pi^{2} \epsilon}\left(\frac{H}{M_{p}}\right)^{2}\right]+\frac{\mathrm{d} \ln \gamma_{S}}{\mathrm{~d} \ln k}$,

and using (28) and (36), the second term in (45) for $\chi \simeq 0.75$ can be written as

$\frac{\mathrm{d} \ln \gamma_{S}}{\mathrm{~d} \ln k} \simeq 2 \cot \varphi_{S} \frac{\mathrm{d} \varphi_{S}}{\mathrm{~d} \ln k}$.

Since $\varphi_{S}$ is an arbitrary parameter, we can assume that $\varphi_{S}$ is $k$ independent. With this choice, the second term in (45), which represents the effects of $\alpha$-vacua, will vanish and in consequence the spectral index remains intact.

\section{Concluding remarks}

In this work we studied the Higgs inflation model under general initial conditions. The general initial conditions affect inflationary observables such as the power spectrum, the non-Gaussianity, etc. The effects of the general initial conditions are constrained by the requirement that they should not spoil the inflationary background. Moreover, the observational bound on the non-Gaussianity of the primordial perturbations gives another constraint on these initial condition effects. We argued that for some region of parameter space in the initial conditions, it is possible to enhance the tensor to scalar perturbation ratio, $r$. This enhancement was possible, because the Higgs inflation scenario gives a very small value for the slow-roll parameter $\epsilon$. The smallness of $\epsilon$ enables us to access a more extended region of parameter space without violation of the observation bound on the non-Gaussianity. A suitable choice of the region of parameter space may lead to a value of $r \simeq 0.05$, which is a desirable value considering the latest results from the BICEP2/Keck Array and Planck collaborations. 
Acknowledgments S. Z would like to thank M. M. Sheikh-Jabbari and M. Golshani for encouragement and support. This work has been supported financially by the Research Institute for Astronomy and Astrophysics of Maragha (RIAAM) under research project "Higgs inflation", Maragha, Iran.

Open Access This article is distributed under the terms of the Creative Commons Attribution 4.0 International License (http://creativecomm ons.org/licenses/by/4.0/), which permits unrestricted use, distribution, and reproduction in any medium, provided you give appropriate credit to the original author(s) and the source, provide a link to the Creative Commons license, and indicate if changes were made.

Funded by SCOAP ${ }^{3}$.

\section{References}

1. A. Linde, arXiv: 1402.0526 [hep-th]

2. P.A.R. Ade et al. [Planck Collaboration], arXiv:1502.02114 [astroph.CO]

3. P.A.R. Ade et al. [BICEP2 Collaboration], Phys. Rev. Lett. 112, 241101 (2014) arXiv:1403.3985 [astro-ph.CO]

4. R. Flauger, J.C. Hill, D.N. Spergel, JCAP 1408, 039 (2014). arXiv:1405.7351 [astro-ph.CO]

5. M.J. Mortonson, U. Seljak, JCAP 1410(10), 035 (2014). arXiv: 1405.5857 [astro-ph.CO]

6. P.A.R. Ade et al. [BICEP2 and Keck Array Collaborations], arXiv: 1502.00643 [astro-ph.CO]

7. F.L. Bezrukov, M. Shaposhnikov, Phys. Lett. B 659, 703 (2008). arXiv:0710.3755 [hep-th]

8. P.A.R. Ade et al. [BICEP2 and Planck Collaborations], Phys. Rev. Lett. 114(10), 101301 (2015). arXiv:1502.00612 [astro-ph.CO]

9. P.A.R. Ade et al. [Planck Collaboration], arXiv:1502.01589 [astroph.CO]

10. J.L.F. Barbon, J.R. Espinosa, Phys. Rev. D 79, 081302 (2009). arXiv:0903.0355 [hep-ph]

11. C.P. Burgess, H.M. Lee, M. Trott, JHEP 1007, 007 (2010). arXiv:1002.2730 [hep-ph]
12. M.P. Hertzberg, JHEP 1011, 023 (2010). arXiv:1002.2995 [hep-ph]

13. C. Germani, A. Kehagias, Phys. Rev. Lett. 105, 011302 (2010). arXiv:1003.2635 [hep-ph]

14. F. Bezrukov, A. Magnin, M. Shaposhnikov, S. Sibiryakov, JHEP 1101, 016 (2011). arXiv:1008.5157 [hep-ph]

15. S. Ferrara, R. Kallosh, A. Linde, A. Marrani, A. Van Proeyen, Phys. Rev. D 83, 025008 (2011). arXiv: 1008.2942 [hep-th]

16. Y. Hamada, H. Kawai, K. y. Oda, S. C. Park. Phys. Rev. Lett. 112, 241301 (2014). arXiv:1403.5043 [hep-ph]

17. P. Ko, W.I. Park, arXiv:1405.1635 [hep-ph]

18. J. Rubio, M. Shaposhnikov, Phys. Rev. D 90, 027307 (2014). arXiv:1406.5182 [hep-ph]

19. Y. Hamada, H. Kawai, K.y. Oda, S.C. Park, arXiv:1408.4864 [hep$\mathrm{ph}]$

20. R. Easther, B.R. Greene, W.H. Kinney, G. Shiu, Phys. Rev. D 64, 103502 (2001). arXiv:hep-th/0104102

21. P.R. Anderson, C. Molina-Paris, E. Mottola, Phys. Rev. D 72, 043515 (2005). arXiv:hep-th/0504134

22. A. Ashoorioon, K. Dimopoulos, M.M. Sheikh-Jabbari, G. Shiu, JCAP 1402, 025 (2014). arXiv:1306.4914 [hep-th]

23. A. Ashoorioon, K. Dimopoulos, M.M. Sheikh-Jabbari, G. Shiu, Phys. Lett. B 737, 98 (2014). arXiv:1403.6099 [hep-th]

24. F. Bezrukov, Class. Quant. Grav. 30, 214001 (2013). arXiv:1307.0708 [hep-ph]

25. P.A.R. Ade et al. [Planck Collaboration], Astron. Astrophys. 571, A16 (2014). arXiv:1303.5076 [astro-ph.CO]

26. D. Baumann, arXiv:0907.5424 [hep-th]

27. D. Boyanovsky, H.J. de Vega, N.G. Sanchez, Phys. Rev. D 74, 123006 (2006). arXiv:astro-ph/0607508

28. S. Kundu, JCAP 1404, 016 (2014). arXiv:1311.1575 [astro-ph.CO]

29. R. Flauger, D. Green, R.A. Porto, JCAP 1308, 032 (2013). arXiv:1303.1430 [hep-th]

30. R. Holman, A.J. Tolley, JCAP 0805, 001 (2008). arXiv:0710.1302 [hep-th]

31. P.A.R. Ade et al. [Planck Collaboration], arXiv:1502.01592 [astroph.CO] 